\title{
Using Machine Learning to Analyze Climate Change TECHNOLOGY TRANSFER (CCTT)
}

\author{
Shruti Kulkarni \\ Department of Management Studies \\ Indian Institute of Science \\ Bangalore, India \\ \{shrutik\}@iisc.ac.in
}

\begin{abstract}
The objective of the present paper is to review the current state of climate change technology transfer. This research proposes a method for analyzing climate change technology transfer using patent analysis and topic modeling. A collection of climate change patent data from patent databases would be used as input to group patents in several relevant topics for climate change mitigation using the topic exploration model in this research. The research questions we want to address are: how have patenting activities changed over time in climate change mitigation related technology (CCMT) patents? And who are the technological leaders? The investigation of these questions can offer the technological landscape in climate change-related technologies at the international level. We propose a hybrid Latent Dirichlet Allocation (LDA) approach for topic modelling and identification of relationships between terms and topics related to CCMT, enabling better visualizations of underlying intellectual property dynamics. Further, a predictive model for CCTT is proposed using techniques such as social network analysis (SNA) and, regression analysis. The competitor analysis is also proposed to identify countries with a similar patent landscape. The projected results are expected to facilitate the transfer process associated with existing and emerging climate change technologies and improve technology cooperation between governments.
\end{abstract}

\section{INTRODUCTION}

The Intergovernmental Panel on Climate Change (IPCC) defines technology transfer (TT) as "a broad set of processes covering the flows of know-how, experience, and equipment for mitigating and adapting to climate change among different stakeholders such as Governments, private sector entities, financial institutions, nongovernmental organizations (NGOs) and research/educational institutions." (Metz et al., 2000). Schnepp et al. (1990) define technology transfer as "A process by which expertise or knowledge related to some aspect of technology is passed from one user to another for the purpose of economic gain". In the case of the transfer of low-carbon technology, the economic benefits that include the mitigation of the future costs associated with climate change as well as any financial benefits to the companies involved in the transfer process. Technology transfer is not only about the exchange of device or know-how across national or international frontiers, but also about the complicated processes of sharing knowledge and adapting technology to meet local conditions.

\subsection{NEED FOR TECHNOLOGY TRANSFER}

Agenda 21 that resulted from the United Nations Conference on Environment and Development recognizes that "there is a need for favorable access to and transfer of environmentally sound technologies, in particular to developing countries, through supportive measures that promote technology cooperation and that should enable transfer of necessary technological know-how as well as building up of economic, technical, and managerial capabilities for the efficient use and further development of transferred technology" (Carroll-Foster, 1993). The Johannesburg Plan of Implementation 
(JPOI) that resulted from the World Summit on Sustainable Development calls upon governments and relevant regional and international organizations to take action on development, dissemination and deployment of affordable cleaner energy, energy efficiency and energy conservation technologies and the transfer of these technologies to developing countries (DSD, 2002). Figure11 highlights intellectual property (IP) landscape in the field of climate change technologies. Drawing upon new extractions from the Worldwide Patent Statistical Database (PATSTAT), The International Energy Agency (IEA) and Organisation for Economic Co-operation and Development (OECD) have found that while patenting of innovations in climate change mitigation technologies (CCMT) related to power generation, transport, buildings, manufacturing, and carbon capture and storage (CCS) had generally been increasing much faster than other technologies in the period up to 2011-2012 (IEA. 2019). However, there has been a notable drop-off in the number of these patents since 2015 as represented by Figure 1

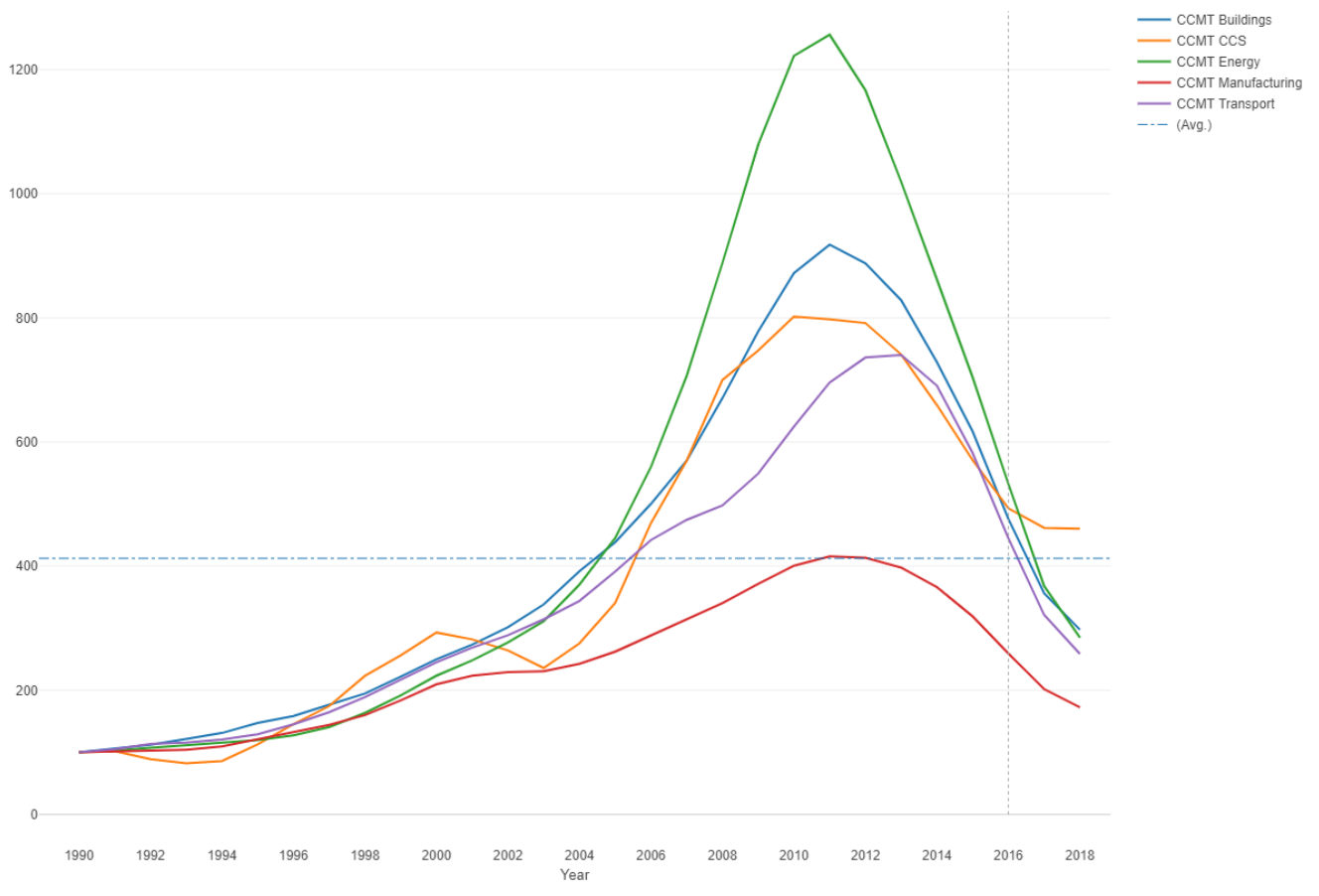

Figure 1: Global patent applications for climate change mitigation technologies 1990-2015.Source: Re-plotted by author based on patent extractions from EPO World Patent Statistical Database (PATSTAT) by OECD/ENV and IEA/EDC(2019).

\section{Methodology}

Studies indicate that most of the technology transfer that takes place is among developed countries (Stewart, 1992). The rate of global patenting on the climate change mitigation technologies is essential for dissemination of knowledge globally. For analyzing climate change patents transfer data we propose a step by step methodology as represented in Figure 2

\subsection{STEP 1: DATA COLLECTION}

The patent documents related to climate change technologies will be collected from the United States Patent and Trademark Office (USPTO)'s online database. The data source is appropriate for exploring technological trends because it is a representative patent database containing an enormous number of patents from all over the world and covers the most advanced technologies (Kim \& Lee, 2015). However, country wise comparisons to the climate change technologies poses a further challenge (Schmoch, 2008). The proposed search query for the data collection consists of terms dealing with climate change mitigation technologies, combined with climate change domain ontology and 


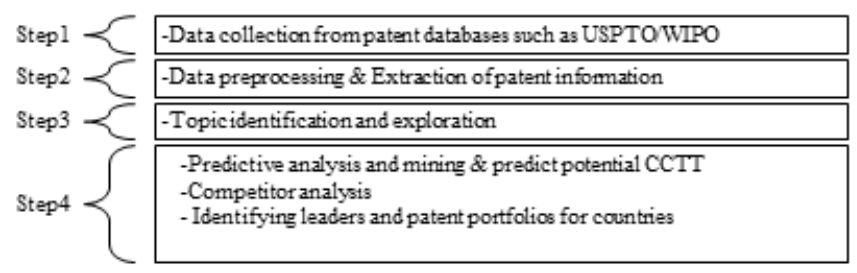

Figure 2: Proposed methodology

domain terms such as biodiversity, carbon, climate, ecology, environment, emission, ICT for climate change mitigation, energy storage, sustainable, etc.

\subsection{STEP 2: DATA PREPROCESSING}

The collected patent documents represent an unstructured text format. Therefore, in step 2 the data would be pre-processed and transformed into a structured format for further analyses. The preprocessing procedure will be performed using the document parsing techniques. The relevant items, such as the title, abstract, assignees, filing year, register year, classification code, and citation will be extracted from documents. For this purpose, the abstract in a free-text format will be required for further pre-processing tasks with natural language processing techniques, including tokenization, lemmatization, stop-word removing, and vector-space representation. Among these text items, the abstract will be used as the input to lda2vec to identify topics because it essentially includes the main problem addressed by the patented technology.

\section{STEP 3: TOPIC IDENTIFICATION AND EXPLORATION}

Figure 3 summarises research design for steps 1 to 3 . Patent data offers rich, contextualized in-
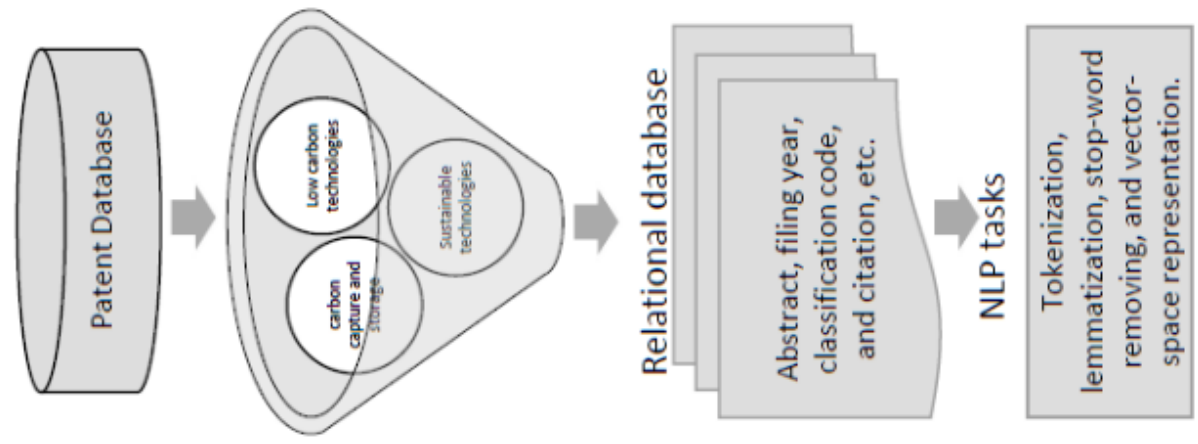

Figure 3: Summary of research design

formation about patent technology domains, inventor countries and their complex connections. To analyze and interpret patent data, we propose the use of topic modeling. Topic modeling is a statistical approach for discovering topics that occur in a document corpus (Blei et al. 2003). The research question we will be addressing by this step is: what is the topic landscape of patents filed for climate change mitigation technologies? We propose a hybrid LDA approach for topic identification and exploration using lda2vec to address the question. Lda2vec (Moody, 2016) combines the power of word2vec (Mikolov et al. 2013) with the interpreability of LDA. Inspired by Latent Dirichlet Allocation (LDA), the word2 vec model is expanded to simultaneously learn word, document and topic vectors. Based on the per-topic distribution, each patent document will be assigned to one of $\mathrm{k}$ topics exhibiting the highest probability. In this step we have the following objectives-

1. Grouping patent documents with similar topic probability distributions; 
2. Increasing the understanding of the latent topic structure by producing a term distribution over each topic;

3. Label the $\mathrm{k}$ identified topics in the climate change mitigation - related patents.

\subsection{STEP 4: FURTHER ROADMAP}

In this last step, the identified topics are further explored from two aspects: trends in patenting activities over time and assignee in each topic. The research questions are defined as follows: how have patenting activities changed over time? and who have been technological leaders in climate change related patents? The investigation of these questions can offer the technological landscape in climate change related technologies at the international level.

After the investigation of patent landscape, we would further like to build predictive models based on our patent analysis for technology transfer. We would like to highlight few prior studies that used predictive modeling based on patent information. Mogee (1991) used patent families to analyze R\&D planning, international patent activity, and patent indicators. Wu et al. (2010) constructed a predictive model based on International Patent Classification (IPC) codes to assess possibilities of patent registration. Jun (2011); Park et al. (2015) studied data mining techniques for technology forecasting using patent information such as title, abstract, IPC codes, and bibliographic data. Kim et al. (2013) proposed a hierarchical analysis model of the decision-making process in order to predict technology transfer policy directions. There are plenty of predictive machine learning algorithms that can be applied to our study. We propose a social network based analysis for this step, because SNA is an efficient approach to analyze the patent data (Jun \& Park, 2013). Using the SNA, we can get the association between patent-based indicators to construct the predictive model for technology transfer for a particular targeted technology.

The patent-based indicators based on IPC codes, inventor country, citation information, and so on will be fetched to SNA graphs. The social network structures may contain a number of nodes consisting of information for a particular targeted technology such as the number of forward citations, novelty, number of backward citations, number of International Patent Documentation (INPADOC) family patents, patent duration (expiration date - registered date), number of forward citations, number of IPC codes extracted, and so on. This research further proposes a regression analysis of the visualization results from SNA graphs. The results from the SNA will be used all together to explore meaningful factors for predictive models. To facilitate the process of technology transfer, we further propose a competitor analysis between countries. It would be very useful for countries to know what the trend of a competitor's technology development is. Based on the topic modeling results, we propose competitor analysis using the following techniques:

- Word-based similarity (WBS): WBS represents countries by a vector of words, and it would rank the competitors based on (Cosine) similarity between countries.

- Topic-based divergence (TBD): It represents each country's patent portfolio using the topic distribution and ranks the competitors by the KL-divergence.

\section{PRojected RESUlts}

In general, the transferred technologies are important nationally and internationally for improving their technological competitiveness. Using the methodology proposed in this study, we aim to give investors, governments and policy makers recommendations based on following projections:

1. Analysis of patent portfolios for disclosed regarding climate change related topics using LDA;

2. Find which countries are addressing the threat of climate change in their patent portfolios;

3. Aid developing countries for capacity building for climate change technology development and transfer;

4. Aid policy makers in creating new programmes such as the Clean Development Mechanism (CDM), AsiaPacific Partnership for positive advances in the case of international technology transfer; 
5. Providing a predictive model of technology transfer by collecting patent data and applying text mining techniques for preprocessing.;

In conclusion, we proposed a model that promotes developed countries to concretely pursue technology transfer with developing countries in the field of climate change related technologies. We hope it would further open up possible domain exploration for technology transfers for climate change adaptation and mitigation.

\section{REFERENCES}

David M Blei, Andrew Y Ng, and Michael I Jordan. Latent dirichlet allocation. Journal of machine Learning research, 3(Jan):993-1022, 2003.

Theodora Carroll-Foster. Transfer of environmentally sound technology, cooperation and capacity building. In Assessment of Agenda 21: toward a national sustainable development strategy for Canada. Projet de Société, Ottawa, ON, CA, 1993.

DSD. Division for Sustainable Development (DSD), Areas of Work: Energy, Intergovernmental Decisions, 2002. https://www.un.org/esa/dsd/dsd_aofw_ene/ ene_integovedeci.shtml.

IEA. International Energy Agency, Global patent applications for climate change mitigation technologies - a key measure of innovation - are trending down, 2019. URL https://www.iea.org/commentaries/global-patent-applicationsfor-climate-change-mitigation-technologies-a-key-measure-ofinnovation-are-trending-down

Sung-Hae Jun. Technology forecasting of intelligent systems using patent analysis. Journal of Korean institute of intelligent Systems, 21(1):100-105, 2011.

Sunghae Jun and Sang Sung Park. Examining technological innovation of apple using patent analysis. Industrial Management \& Data Systems, 2013.

HM Kim, JH Han, and YB Kim. Study on future foresight of the technology commercialization policy. The Journal of Industrial Economics and Business, 26(2):803-824, 2013.

Jeeeun Kim and Sungjoo Lee. Patent databases for innovation studies: A comparative analysis of USPTO, EPO, JPO and KIPO. Technological Forecasting and Social Change, 92:332-345, 2015.

Bert Metz, Ogunlade R Davidson, John K Turkson, Jan-Willem Martens, Sascha NM van Rooijen, and Laura van Wie McGrory. Methodological and technological issues in technology transfer: a special report of the intergovernmental panel on climate change. Cambridge University Press, 2000.

Tomas Mikolov, Ilya Sutskever, Kai Chen, Greg S Corrado, and Jeff Dean. Distributed representations of words and phrases and their compositionality. In Advances in neural information processing systems, pp. 3111-3119, 2013.

Mary Ellen Mogee. Using patent data for technology analysis and planning. Research-Technology Management, 34(4):43-49, 1991.

Christopher E Moody. Mixing dirichlet topic models and word embeddings to make lda2vec. arXiv preprint arXiv:1605.02019, 2016.

Sangsung Park, Seung-Joo Lee, and Sunghae Jun. A network analysis model for selecting sustainable technology. Sustainability, 7(10):13126-13141, 2015.

Ulrich Schmoch. Concept of a technology classification for country comparisons: Final report to the World Intellectual Property Organisation (WIPO). Fraunhofer Institute for Systems and Innovation Research, Karlsruhe, 63, 2008.

Otto Schnepp, Mary Ann Young Von Glinow, and Arvind Bhambri. United States-China Technology Transfer. Prentice Hall, 1990. 
Frances Stewart. Technology transfer for development. In North-South and South-South, pp. 311338. Springer, 1992.

Chih-Hung $\mathrm{Wu}$, Yun Ken, and Tao Huang. Patent classification system using a new hybrid genetic algorithm support vector machine. Applied Soft Computing, 10(4):1164-1177, 2010. 